\title{
Electrical Stress on Irradiated Thin Gate Oxide Partially Depleted SOI nMOSFETs
}

\author{
J. M. Rafí a, ${ }^{,}$, E. Simoen ${ }^{b}$, A. Mercha ${ }^{b}$, K. Hayama ${ }^{c}$, \\ F. Campabadal ${ }^{\mathrm{a}}, \mathrm{H}$. Ohyama ${ }^{\mathrm{c}}$, C. Claeys ${ }^{\mathrm{b}, \mathrm{d}}$ \\ ${ }^{a}$ Centro Nacional de Microelectrónica (IMB-CNM-CSIC), Campus UAB, 08193 Bellaterra (Barcelona), Spain \\ ${ }^{b} I M E C$, Kapeldreef 75, B-3001 Leuven, Belgium \\ ${ }^{c}$ Kumamoto National College of Technology, 2569-2 Nishigoshi, Kumamoto 861-1102, Japan \\ ${ }^{d}$ Katholieke Universiteit Leuven, Dept. of Electrical Engineering, Kasteelpark Arenberg 10, B-3001 Leuven, Belgium
}

\begin{abstract}
The effect of hot-carrier stress on $60 \mathrm{MeV}$ proton irradiated thin gate oxide partially depleted SOI nMOSFETs has been studied. The results are compared with those from the electrical stress of non-irradiated devices. Whereas no significant differences are observed for the front channel degradation, hot-electron trapping in the buried oxide is found to be enhanced in the irradiated devices. This hot-electron trapping leads to a compensation or neutralization of the effects caused by the radiation-induced positive trapped charges. It is shown that a similar hot-electron trapping enhancement can be achieved in non-irradiated devices stressed under certain back gate bias conditions.
\end{abstract}

Keywords: Silicon on insulator; Partially-depleted SOI; Hot-carrier degradation; Radiation-induced damage; MOSFETs

\section{Introduction}

SOI technologies are promising candidates for the continued scaling of transistor size [1]. However, the presence of the thick buried oxide (BOX) may introduce some constraints. It is well-known that they can suffer from radiation-induced positive charge trapping in the BOX [2-4]. Moreover, in the case of

\footnotetext{
* Corresponding author. Tel.:+ 3493 5947700; fax: +3493 5801496.

E-mail address:jmrafi@cnm.es (J. M. Rafí)
}

hot-carrier (HC) degradation, opposite channel injection phenomena may occur [5]. The consequences increase as the silicon film thickness is reduced and gate coupling arises.

In this contribution, the effect of hot-carrier stress on $60 \mathrm{MeV}$ proton irradiated thin gate oxide partially depleted (PD) SOI nMOSFETs is studied. The results are compared with those from the electrical stress of non-irradiated devices. The interaction and similarities between the radiation damage and the one generated during electrical stress at different conditions are studied. 


\section{Experimental}

The PD SOI nMOSFETs used in this study were fabricated in a $0.1 \mu \mathrm{m}$-CMOS process using PELOX isolation and a $2.5 \mathrm{~nm}$ nitrided gate oxide. The BOX and film thickness were $400 \mathrm{~nm}$ and $100 \mathrm{~nm}$, respectively. Isolated transistors with polysilicon gate lengths (L) ranging from $0.08 \mu \mathrm{m}$ to $10 \mu \mathrm{m}$ and fixed width $(\mathrm{W}=10 \mu \mathrm{m})$ were used for this work. The devices had no film contact. Some samples were subjected to unbiased $60 \mathrm{MeV}$ proton irradiations at the Cyclone cyclotron facility (Louvain-la-Neuve, Belgium). A fluence of $2 \cdot 10^{11}$ protons $/ \mathrm{cm}^{2}$ was used, which corresponds to a dose suitable for space applications assessment. For the hot-carrier degradation (HCD) analysis, both, irradiated and non-irradiated transistors, were stressed at constantvoltage stress under different drain voltages $\left(\mathrm{V}_{\mathrm{D}}\right)$ and at the worst-case front gate voltage $\left(\mathrm{V}_{\mathrm{FG}}\right)$ regime for the HCD of these devices $\left(\mathrm{V}_{\mathrm{FG}}=\mathrm{V}_{\mathrm{D}}\right)$ [6]. Front and back channel characteristics were regularly measured between stress phases and their respective threshold voltages $\left(\mathrm{V}_{\mathrm{Tf}}\right.$ and $\left.\mathrm{V}_{\mathrm{Tb}}\right)$ were extracted. Unless otherwise is indicated, the back gate was grounded $\left(\mathrm{V}_{\mathrm{BG}}=0 \mathrm{~V}\right)$ and $\mathrm{V}_{\mathrm{D}}$ was kept at $25 \mathrm{mV}$.

\section{Irradiation+HCD: front channel characteristics}

Figure 1 shows typical $\mathrm{I}_{\mathrm{D}}-\mathrm{V}_{\mathrm{FG}}$ characteristics for a non-irradiated nMOSFET, as well as its characteristics after $2 \cdot 10^{11}$ protons $/ \mathrm{cm}^{2}$ irradiation. An increase in the subthreshold leakage current (hump) is observed after irradiation, which is originated by the presence of radiation-induced positive trapped charges in the thick BOX, giving rise to a back channel parasitic conduction [7]. This radiation-induced $I_{D}$ subthreshold leakage can be suppressed by applying a sufficiently negative $\mathrm{V}_{\mathrm{BG}}$, which keeps the back channel into accumulation [7]. However, in this work, it is shown that the radiationinduced subthreshold leakage can be progressively eliminated by the HCD of such irradiated devices (Fig. 1). Regarding the portion of the characteristics above $\mathrm{V}_{\mathrm{Tf}}$, no significant $\mathrm{HCD}$ differences have been observed between irradiated and non-irradiated devices. The degradation is initially dominated by a positive shift of $\mathrm{V}_{\mathrm{T} f}$, with a turn-around behavior for sufficiently longer/harder stress conditions (Fig. 2) $[6,8]$.

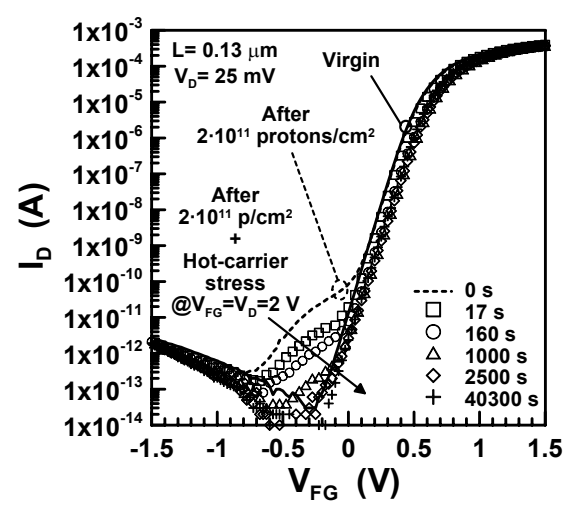

Fig. 1: $I_{D}$ vs. $V_{F G}$ for an $L=0.13 \mu \mathrm{m}$ non-irradiated (virgin) nMOSFET, after $2 \cdot 10^{11}$ protons $/ \mathrm{cm}^{2}$ irradiation and after different subsequent $\mathrm{HC}$ stress times at $\mathrm{V}_{\mathrm{FG}}=\mathrm{V}_{\mathrm{D}}=2 \mathrm{~V}$.

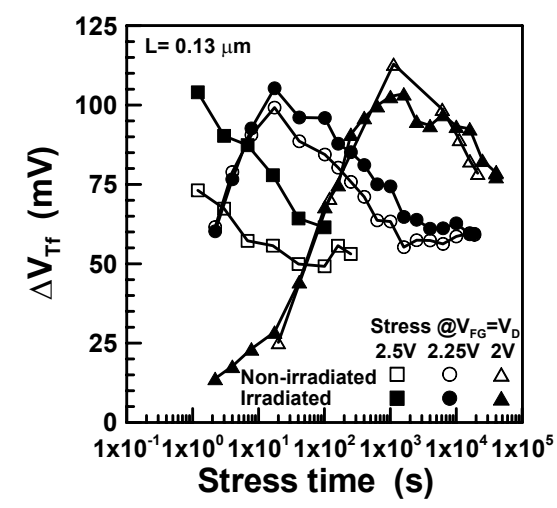

Fig. 2: Front gate threshold voltage shift $\left(\Delta \mathrm{V}_{\mathrm{Tf}}\right)$, versus hotcarrier stress time for different non-irradiated and irradiated nMOSFETs stressed under $\mathrm{V}_{\mathrm{FG}}=\mathrm{V}_{\mathrm{D}}$ conditions.

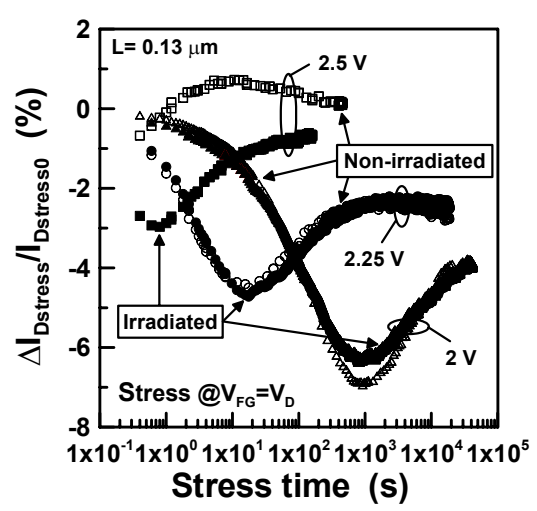

Fig. 3: Degradation of $I_{D}$ values measured during stress for Fig. 2 nMOSFETs. (Where $\Delta \mathrm{I}_{\text {Dstress }}=\mathrm{I}_{\text {Dstress }}-\mathrm{I}_{\text {Dstress0 } 0}$ and $I_{\text {Dstress0 }}$ is the $I_{\text {Dstress }}$ value at the beginning of the HC stress). 
Occasionally the degradation of the front channel characteristics has been found to be a bit larger in the case of the irradiated devices, but this is similarly accelerated for reduced $\mathrm{L}$ and increased stress bias conditions (Figs. 2 and 3). Regarding the impact on the $I_{D}$ hysteresis that can be observed in these floating body devices, apart from the observed $\mathrm{V}_{\mathrm{Tf}}$ shift and $\mathrm{I}_{\mathrm{D}}$ hysteresis peaks degradation associated with the HCD [9], the only difference between irradiated and non-irradiated devices is the existence of an initial subthreshold $\mathrm{I}_{\mathrm{D}}$ hysteresis in the case of the irradiated devices, which gradually vanishes with the progress of the HC stress (Fig. 4).

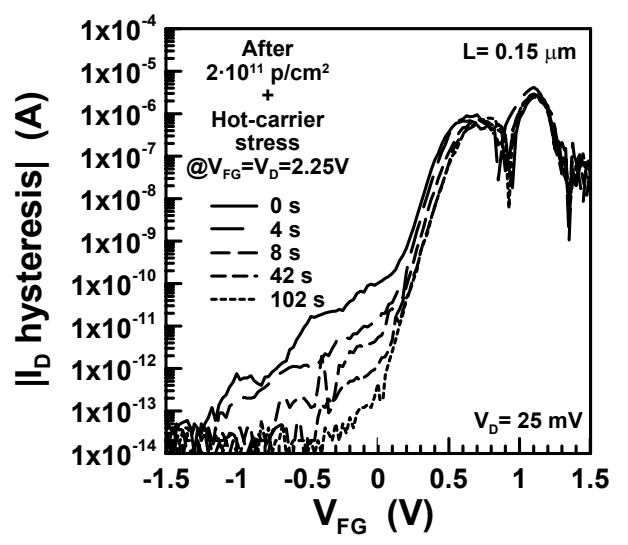

Fig. 4: $\mid I_{D}$ hysteresis $\mid\left(I_{D}\right.$ difference between accumulation to inversion and inversion to accumulation $\mathrm{V}_{\mathrm{FG}}$ sweeps) for an irradiated nMOSFET before HCD and after different stress times.

\section{Irradiation+HCD: back channel characteristics}

Interestingly, the degradation of the back channel characteristics is characterized by a significant positive $\mathrm{V}_{\mathrm{Tb}}$ shift (Fig. 5), which is found to be enhanced in the presence of the radiation damage (Fig. 6). In this way, the effect of the radiationinduced positive trapped charges is neutralized or compensated by the trapping of hot-electrons in the thick buried oxide (Fig. 7). In the past, a minimal interaction between hot-carrier and X-ray radiation effects was reported for pMOSFETs of a $0.3 \mu \mathrm{m}$ fully depleted SOI technology [3]. On the other hand, a higher sensitivity of the BOX to hot-carrier aging was observed in nMOSFETs using SIMOX substrates when the transistors were subjected to back channel hot-carrier stress [2].

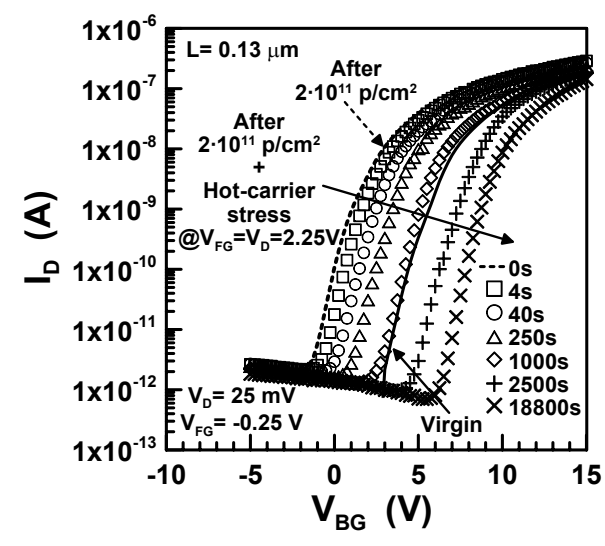

Fig. 5: $I_{D}$ vs. $V_{B G}$ for an $L=0.13 \mu \mathrm{m}$ non-irradiated (virgin) nMOSFET, after $2 \cdot 10^{11}$ protons $/ \mathrm{cm}^{2}$ irradiation and after different subsequent $\mathrm{HC}$ stress times at $\mathrm{V}_{\mathrm{FG}}=\mathrm{V}_{\mathrm{D}}=2.25 \mathrm{~V}$.

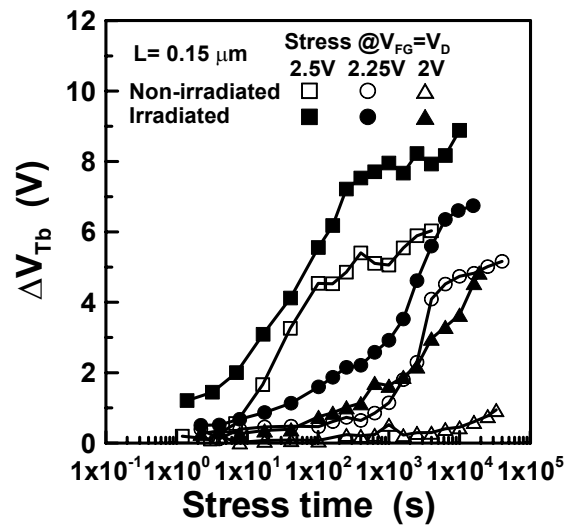

Fig. 6: Back gate threshold voltage shift $\left(\Delta \mathrm{V}_{\mathrm{Tb}}\right)$ versus hotcarrier stress time for different irradiated and non-irradiated nMOSFETs stressed under $\mathrm{V}_{\mathrm{FG}}=\mathrm{V}_{\mathrm{D}}$ conditions.

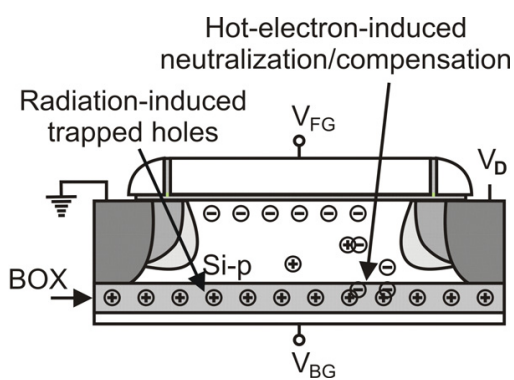

Fig. 7: Sketch of a PD SOI nMOSFET showing radiationinduced trapped holes in the BOX and subsequent hotelectron trapping, leading to a neutralization or compensation of the radiation-induced damage effects. 


\section{HCD at different back gate bias conditions}

HCD experiments carried out under worst-case $\mathrm{V}_{\mathrm{FG}}=\mathrm{V}_{\mathrm{D}}$ regime and different $\mathrm{V}_{\mathrm{BG}}$ have also revealed an enhanced BOX hot-electron trapping under $\mathrm{V}_{\mathrm{BG}}>0$ conditions (Fig. 8). It is inferred that the application of a positive $\mathrm{V}_{\mathrm{BG}}$ in non-irradiated devices, or, similarly, the presence of radiation-induced trapped holes in the BOX, can enhance the hot-electron trapping. However, as in the case of the irradiated devices (Figs. 2 and 3), no appreciable $\mathrm{V}_{\mathrm{BG}}$-induced changes are observed for the degradation of the front channel characteristics (Fig. 8). Finally, the results from additional HCD experiments carried out under $\mathrm{V}_{\mathrm{FG}} \approx \mathrm{V}_{\mathrm{Tf}}$ regime have shown that a different sign for the BOX trapped charges can be achieved depending on the $\mathrm{V}_{\mathrm{BG}}$ applied during the $\mathrm{HC}$ stress (Fig. 9).

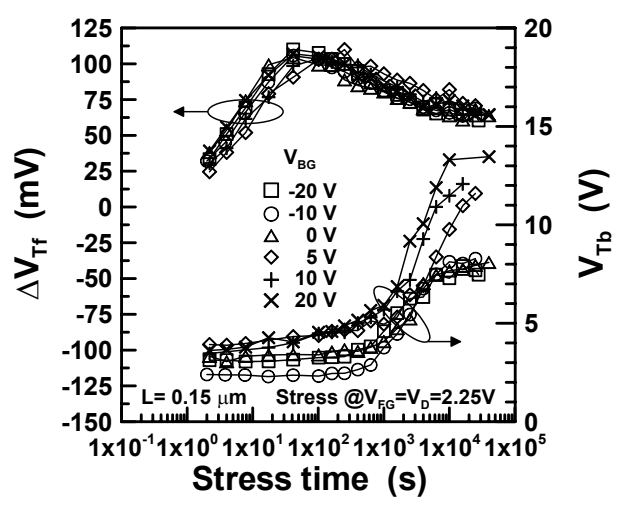

Fig. 8: Degradation of $\mathrm{V}_{\mathrm{Tf}}$ and $\mathrm{V}_{\mathrm{Tb}}$ for different nonirradiated nMOSFETs stressed under a fixed $\mathrm{V}_{\mathrm{FG}}=\mathrm{V}_{\mathrm{D}}=2.25$ $\mathrm{V}$ condition and different $\mathrm{V}_{\mathrm{BG}}$ biases.

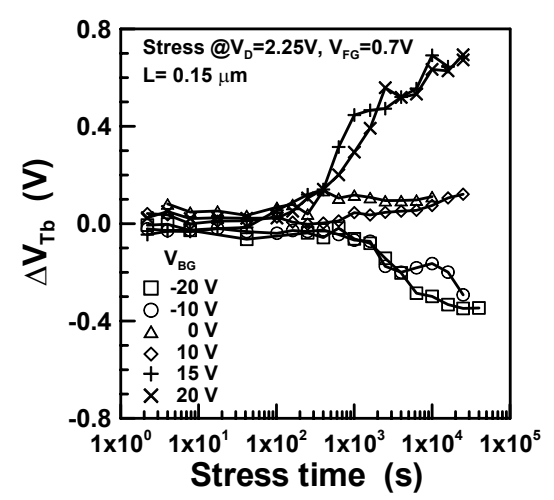

Fig. 9: $\Delta \mathrm{V}_{\mathrm{Tb}}$ for different non-irradiated nMOSFETs stressed under a fixed $\mathrm{V}_{\mathrm{D}}=2.25 \mathrm{~V}$ and low $\mathrm{V}_{\mathrm{FG}}$ regime $\left(\mathrm{V}_{\mathrm{FG}}=0.7 \mathrm{~V}\right)$ condition and different $\mathrm{V}_{\mathrm{BG}}$ biases.

\section{Conclusions}

The effect of $\mathrm{HC}$ stress on $60 \mathrm{MeV}$ proton irradiated PD SOI nMOSFETs has been studied. Whereas no significant differences are observed for the front channel degradation, hot-electron trapping in the BOX is found to be enhanced in irradiated devices. This leads to a compensation of the effects caused by the radiation-induced trapped charges. It is shown that a similar hot-electron trapping enhancement can be achieved in non-irradiated devices stressed under certain $\mathrm{V}_{\mathrm{BG}}$ conditions. The degradation of the thick BOX can introduce some constraints in emerging SOI technologies.

\section{Acknowledgements}

This work has been partially supported by Ramón y Cajal program of the Spanish Ministry of Science.

\section{References}

[1] Int. Tech. Roadmap for Semiconductors, 2006 update.

[2] T. Ouisse, S. Cristoloveanu, and G. Borel, Electron trapping in irradiated SIMOX buried oxides, IEEE Electron Dev. Lett. 12 (1991) 312-314.

[3] H.-F. Wei, J.E. Chung, and N.K. Annamalai, Buriedoxide charge trapping induced performance degradation in FD ultra-thin SOI p-MOSFET's, IEEE Trans. Electron Dev. 43 (1996) 1200-1205.

[4] J.R. Schwank, V. Ferlet-Cavrois, M.R. Shaneyfelt, and P.E. Dodd, Radiation effects in SOI technologies, IEEE Trans. Nucl. Sci. 50 (2003) 522-538.

[5] D.E. Ioannou, F.L. Duan, S.P. Sinha, and A. Zaleski, Opposite-channel-based injection of hot-carriers in SOI MOSFET's: physics and applications, IEEE Trans. Electron Dev. 45 (1998) 1147-1154.

[6] J.M. Rafí, E. Simoen, A. Mercha, F. Campabadal, and C. Claeys, Impact of hot-carrier stress on gate-induced floating body effects and drain current transients of thin gate oxide partially depleted SOI nMOSFETs, Solid-State Electron. 49 (2005) 1536-1546.

[7] J.M. Rafí, A. Mercha, E. Simoen, C. Claeys, and A. Mohammadzadeh, Radiation-induced back channel leakage in $60 \mathrm{MeV}$-proton-irradiated $0.10 \mu \mathrm{m}$-CMOS partially depleted SOI MOSFETs, in: Proc. RADECS (2003) 425-432.

[8] A. Cester, S. Gerardin, A. Paccagnella, E. Simoen, and C. Claeys, Electrical stresses on ultra-thin gate oxide SOI MOSFETs after irradiation, IEEE Trans. Nucl. Sci. 52 (2005) 2252-2258.

[9] J.M. Rafí, E. Simoen, K. Hayama, A. Mercha, F. Campabadal, H. Ohyama, et al., Hot-carrier-induced degradation of drain current hysteresis and transients in thin gate oxide floating body partially depleted SOI nMOSFETs, Microel. Reliab. 46 (2006) 1657-1663. 